\title{
2014 Manuscript Reviewers
}

The following individuals generously offered their time and expertise as manuscript reviewers on one or more occasions between 13 November 2013 and 16 November 2014. We greatly appreciate their assistance.

Abele, F.

Adams, L.

Aitken, A.E.

Alignier, A.

Alisauskas, R.T.

Atkinson, D.E.

Ayles, G.B.

Babaluk, J.A.

Barnhardt, R.J.

Barr, S.

Barr, W.

Bechshoft, T.

Birks, H.J.B.

Bjerregaard, P.

Blinnikov, M.S.

Bond, A.

Bowman, J.S.

Brimelow, J.C.

Brown, C.

Brown, R.D.

Brunel, T.

Bunbury, J.

Côté, S.

Caine, K.J.

Cairns, D.M.

Cairns, S.D.

Castleden, H.

Citta, J.J.

Clarke, J.T.

Coakley, B.

Cotter, R.C.

Cumming, S.

Cybulski, J.S.

Darwent, C.M.

Dawson, P.C.

Diamond, A.W.

Domine, F.

Duhaime, G.

Elmhagen, B.

Elzinga, A.

Falconer, R.K.H.

Fall, J.A.

Ferguson, S.H.

Festa-Bianchet, M.

Forbes, B.C.

Forbes, D.L.

Fox, A.D.

\author{
Franke, A. \\ Frideres, J.S. \\ Friesen, T.M. \\ Fryer, P. \\ Fuglei, E. \\ Gearheard, S. \\ Gibbs, A. \\ Gould, J. \\ Grønnow, B. \\ Gratto-Trevor, C. \\ Gunn, A. \\ Hansen, R.G. \\ Harper, K.A. \\ Harwood, L.A. \\ Healey, G.K. \\ Hegel, T. \\ Heinrichs, T. \\ Henry, A. \\ Herbert, D.J. \\ Herman-Mercer, N. \\ Holtmeier, F.-K. \\ Hooper, R.G. \\ Horstmann-Dehn, L. \\ Houston, C.S. \\ Howe, B. \\ Howell, S.E.L. \\ Huntington, H.P. \\ Issaluk, M.D. \\ Jaakkola, L.M. \\ Jehl, J.R., Jr. \\ Johnson, K.R. \\ Joly, K. \\ Jorgenson, M.T. \\ Kääb, A. \\ Kampp, K. \\ Kellett, D.K. \\ Kendrick, A. \\ Key, J. \\ Kingsley, M.C.S. \\ Kokelj, S.V. \\ Krebs, C.J. \\ Krupnik, I. \\ Laidre, K. \\ Lanctot, $\mathrm{R}$. \\ Lane, R. \\ Lantz, T.C. \\ Larsen, C.V.L.
}

Larsen, J.R.

Larter, N.C.

Lee, D.S.

Loring, E.

Loring, P.

Loseto, L.L.

Lowry, L.F.

Luedecke, C.

Macdonald, S.E.

Mallory, M.L.

Marino, E.

Mays, S.

McGrady, M.J.

Mecklenburg, C.W.

Merbs, C.F.

Mermoz, S.

Metz, M.C.

Meyer, K.

Miller, I.M.

Mitchell, M.

Mochnacz, N.J.

Mortensen, P.

Mosher, D.C.

Muir, D.C.G.

Mullan, S.

Mulrennan, M.E.

Natcher, D.C.

Nesje, A.

Nilsson, L.M.

Nishi, J.S.

Norén, K.

Nymand, J.

O'Faircheallaigh, C.

Pagano, A.

Paolisso, M.J.

Park, R.W.

Parkinson, A.J.

Perren, B.B.

Polfus, J.

Pomerleau, C.

Poole, K.G.

Potter, R.A.

Powell, A.N.

Quakenbush, L.T.

Raper, D.J.

Reichard, S.E.

Richling, B.E.
Riecken, $\mathrm{T}$.

Robertson, G.J.

Ross, S.

Ryan, K.

Schaefer, N.D.

Schieck, J.

Schledermann, P.

Schmidt, J.W., Jr.

Schmutz, J.K.

Scholik-Schlomer, A.

Schuster, P.

Seppä, H.

Shank, C.C.

Sharp, M.J.

Shaw, J.

Skarin, A.

Southcott, C.

Spence, C.

Spindel, R.C.

Stammler, F.

Starkweather, S.

Stenton, D.R.

Symons, C.

Szeto, K.

Talarico, D.

Tegowski, J.

Thode, A.

Thorpe, N.

Tivy, A.

Tyler, N.J.

Usher, P.J.

Vetter, M.A.

Villano Spellman, K.

Villeneuve, P.J.

Warnock, N.

Wenzel, G.W.

Wheate, R.

White, G.

Woodcock, T.

Worster, M.G.

Wurtz, T.

Yackel, J.

Yang, D.

Yates, C.

Zazula, G.D.

Zdanowicz, C. 